\title{
Retinal vascular caliber changes after laser photocoagulation in diabetic retinopathy
}

\author{
Habibah Setyawati Muhiddin, ${ }^{1}$ Idayani Panggalo, ${ }^{1}$ Andi Muhammad Ichsan, ${ }^{1}$ Budu, ${ }^{1}$ Emanuele Trucco, ${ }^{2}$ John Ellis ${ }^{3}$
}

Check for updates

pISSN: 0853-1773 - elSSN: 2252-8083 https://doi.org/10.13181/mji.oa.203806 Med J Indones. 2020;29:366-71

Received: May 29, 2019

Accepted: September 09, 2020

Authors' affiliations:

${ }^{1}$ Department of Ophthalmology, Faculty of Medicine, Universitas Hasanuddin, Makassar, Indonesia, 'VAMPIRE Project, Computing (SSEN), University of Dundee, Dundee, United Kingdom, ${ }^{3}$ Ninewells NHS Hospital and Medical School, Dundee, United Kingdom

\section{Corresponding author:}

Idayani Panggalo

Department of Ophthalmology, Faculty

of Medicine, Universitas Hasanuddin,

Jalan Perintis Kemerdekaan KM. 10,

Tamalanrea Indah, Tamalanrea, Makassar

90245, Indonesia

Tel/Fax: +62-411-580678

E-mail: idayani2k@gmail.com

\begin{abstract}
BACKGROUND Diabetic retinopathy causes vascular dilatation caused by hypoxia, whereas oxygen tension improvement leads to retinal vessels narrowing. Given that laser photocoagulation aims to increase the oxygen tension in the retina, we hypothesized that the narrowing of vessel caliber after the treatment could be possibly demonstrated. This study aimed to assess the changes in the caliber of retinal vessels before and after laser photocoagulation in diabetic retinopathy.
\end{abstract}

METHODS This research was a prospective cohort study on the treatment of diabetic retinopathy by laser photocoagulation, and it was conducted at Universitas Hasanuddin Hospital, Makassar, Indonesia between November 2017-April 2018. Retinal vascular caliber changes were analyzed before and 6-8 weeks after photocoagulation in 30 diabetic eyes. Central retinal arteriolar equivalent (CRAE) and central retinal venular equivalent (CRVE) were measured using the vessel assessment and measurement platform software for images of the retina (VAMPIRE) manual annotation tool.

RESULTS A significant decrease of CRVE was observed after laser photocoagulation $(p<0.001)$, but CRAE was not reduced significantly $(p=0.067)$. No difference was recorded between CRVE and CRAE post-laser photocoagulation $(p=0.14)$, implying a reduction in vein caliber toward normal in the treated eyes.

CONCLUSIONS Laser photocoagulation decreases the CRVE in diabetic retinopathy despite the absence of changes in the grade of diabetic retinopathy.

KEYWORDS diabetic retinopathy, laser photocoagulation, retinal vascular caliber
Retinal blood vessel caliber measured in vivo offers a surrogate for retinopathy severity, which reflects the duration and severity of dysglycemia. ${ }^{1}$ A large venular caliber is directly associated with severe retinopathy. According to the pathophysiological models for diabetic retinopathy, chronic hyperglycemia causes abnormal blood flow and pathological changes in retinal microvascular structures. The earliest changes are represented by thickening of the capillary basement membrane and the loss of pericytes. Rheological changes include an increased plasma viscosity and a decreased capillary blood flow velocity. These changes contribute to the increased capillary permeability, damage to the inner bloodretinal barrier, and ultimately to capillary occlusion and microaneurysm formation. These conditions cause hypoxia in the retina and retinal ischemia, which is the key step in the pathogenesis of diabetic retinopathy main complications, the development of retinal neovascularization, and retinal detachment from massive fibrovascular proliferation or visual loss from vitreous hemorrhage. ${ }^{2}$ Studies revealed the caliber changes from arteriolar and venular retina, which describe different pathophysiological 
processes. ${ }^{1}$ Meanwhile, retinal arteriolar narrowing is associated with diabetes and coronal artery disease. Venular dilation is associated with the development and progression of diabetic retinopathy and stroke. ${ }^{1,3,4}$ Laser photocoagulation aims to reduce the oxygen demand of the retina for ischemic changes in diabetic retinopathy. ${ }^{5}$ After laser photocoagulation, oxygen flow increases from the choroid to the inner retina, causing the constriction of retinal blood vessels. This hypothesis is supported by the possibility that oxygen tension level plays an important role in the autoregulation of retinal blood vessels, in which arterioles dilate when oxygen decreases and constrict when oxygen tension increases. ${ }^{6}$

Photographic techniques and image analysis allow the objective examination of the size of blood vessels in the retina. Retinal image is a potential diagnostic tool because it facilitates the assessment of vascular changes in an accessible and non-invasive manner. Retinal image analysis is one of the active research fields that aims to provide computer assistance methods to help in the measurement and visualization of retinal biomarkers. Blood vessel abnormalities may predict the earliest stages of diabetic retinopathy. Among the several characteristics associated with changes in blood vessels, the central retinal arteriolar equivalent (CRAE) and central retinal venular equivalent (CRVE) have generally been used as indicators for early detection, diagnosis, and staging. ${ }^{2,7}$ In this study, vessel assessment and measurement platform software for images of the retina (VAMPIRE) was used as a tool for the efficient quantification of features of the retinal vasculature with hundreds or thousands of images. VAMPIRE provides visual feedback of the features extracted and a set of tools that allows the user to easily identify, locate, and correct wrong measurements. ${ }^{8,9}$ Several studies used these tools to investigate retinal vascular conditions. ${ }^{10-14}$ Based on the above knowledge, this study aimed to assess changes in the caliber of retinal vessels before and after laser photocoagulation in diabetic retinopathy.

\section{METHODS}

This research was a prospective cohort study on the treatment of diabetic retinopathy by laser photocoagulation and was conducted at Universitas Hasanuddin Hospital, Makassar, Indonesia between November 2017-April 2018. The study applied the principles of the Declaration of Helsinki and approved by the Ethical Committee of Universitas Hasanuddin (No: 32/H4.8.4.5.31/PP36-KOMETIK/2018).

The study enrolled 30 eyes from 23 patients recruited by consecutive sampling. The inclusion criteria were type 2 diabetes mellitus patients with diabetic retinopathy grade of severity: severe nonproliferative diabetic retinopathy and proliferative diabetic retinopathy without clinically significant macular edema, aged between 40-80 years old, agreed to be part of the study, and can provide signed consent and agreed to return for postoperative photographic follow up at 6-8 weeks. The exclusion criteria were other macular abnormalities, such as age-related macular degeneration, epiretinal membranes, glaucoma, keratopathy prevented image acquisition, history of retinal vascular disease, history of ocular trauma, history of hypertension, history of cardiovascular disease, and uncooperative patients. Controls (37 eyes in 23 patients) were selected from their attendance at eye clinics with inclusion criteria of unrelated pathologies, no history of diabetes, and diabetes were specifically excluded with routine blood glucose in clinic, mild-moderate cataract, and no history of hypertension and cardiovascular disease. All subjects underwent complete ocular examination, which included measurements of best-corrected Snellen acuity, afferent pupillary reflex, anterior segment examination (Topcon SL-D7 Digital-Ready Slit Lamp, Topcon Medical Systems, USA), intraocular pressure by non-contact tonometry, and funduscopy with 78-9o D lens (Volk Optical, USA). Glucose control was defined poor if the patients did not take antidiabetic medication regularly, not visit regularly any health care services, had no record of blood sugar monitoring (verbal history taking); meanwhile it was defined good if the patients had visit regularly for their healths and blood glucose level were within normal limits.

Laser photocoagulation was performed using Pascal photocoagulation (OptiMedica, USA) and conducted in all quadrant once per eye. Fundus photographs were obtained using the Topcon TRC50DX (Topcon Medical Systems) fundus camera at baseline and 6-8 weeks post-laser photocoagulation. The images were then used to determine changes in the caliber of venous and arterial vessels. The vascular diameter was measured from the fundus photo through a semi-automatic retinal vascular 
analysis computer program (VAMPIRE, University of Dundee, University of Edinburgh, UK). A posterior pole photograph including the optic nerve and macula was obtained. The VAMPIRE program uses the optic nerve circumference as a referent for determining measurements obtained. The program offers an automated best fit, and the grader/researcher assesses whether this fit is precise enough and has the option to manually improve the "fit" by dragging the boundary marker to the true circumference. The grader then defines vein and artery for the program. The caliber of both blood vessels was defined in zones from 0.5 to 1.0 disk diameter from the optical disk margin. This condition provides a single annotation statistic which is then converted to in vivo vessel diameter in microns using a proprietary formula. ${ }^{15}$ This measurement is then summarized as the average CRAE and CRVE.

Statistical analyses were performed by calculation of range, mean, median, standard deviation, and percentage. Kolmogorov-Smirnov test was used to determine the homogeneity of data distribution. Paired t-test was used to compare CRVE before and after laser and unpaired $t$-test was used to compare CRVE after and before laser with the CRVE control. Mann-Whitney test was used to compare CRAE before and after laser with control and Wilcoxon test was used to compare CRAE before and after laser treatment. Statistical results were examined with SPSS software version 22 (IBM Corp., USA). Microsoft Word and
Excel (Microsoft, USA) were used to generate graphs and tables. $p<0.05$ was considered to be statistically significant.

\section{RESULTS}

A total of 110 eyes of 70 patients obtained by consecutive sampling met the inclusion criteria for the initial and pre-treatment image capture. However, poor results were observed with loss to follow-up and return for post-laser image capture. The reasons for this finding are complex in this population and relate to geography, educational status, income, previous experience of health care, and issues of trust and compliance and lack of awareness. The data presented in this study were 30 eyes of 23 patients who completed post-laser follow-up. Subjects included 12 men and 11 women, and the average age of patients was 57 years.

Assessment of quality of control was problematic in this largely unscreened population sample. Therefore, relatively simple measures of compliance (verbal history taking) were used to assess the glucose control. Table 1 shows the demographic characteristics among subjects.

Figure 1a shows the outcome of the treatment based on CRVE. A significant decrease was observed in CRVE after laser photocoagulation ( $p<0.001)$. CRVE in diabetic retinopathy patients was significantly
Table 1. Demographic characteristics of subject

\begin{tabular}{lcc}
\hline Characteristic & Case, $\mathrm{n}(\%)(\mathrm{N}=23)$ & Control, $\mathrm{n}(\%)(\mathrm{N}=37)$ \\
\hline Male sex & $12(52)$ & $22(59)$ \\
Age (years) & $2(9)$ & $3(8)$ \\
$>30$ & $3(13)$ & $11(30)$ \\
$>40$ & $7(30)$ & $23(62)$ \\
$>50$ & $11(48)$ & \\
$>60$ & & \\
Diabetes duration (years) & $15(65)$ & \\
$<10$ & $8(35)$ & \\
$>10$ & $12(40)$ & \\
Diagnose of diabetic retinopathy & $18(60)$ & \\
\hline Severe NPDR & $11(48)$ & \\
\hline PDR & $12(52)$ & \\
Glucose control & \\
\hline Good & \\
\hline Poor &
\end{tabular}

NPDR=non-proliferative diabetic retinopathy; PDR=proliferative diabetic retinopathy

mji.ui.ac.id 
a

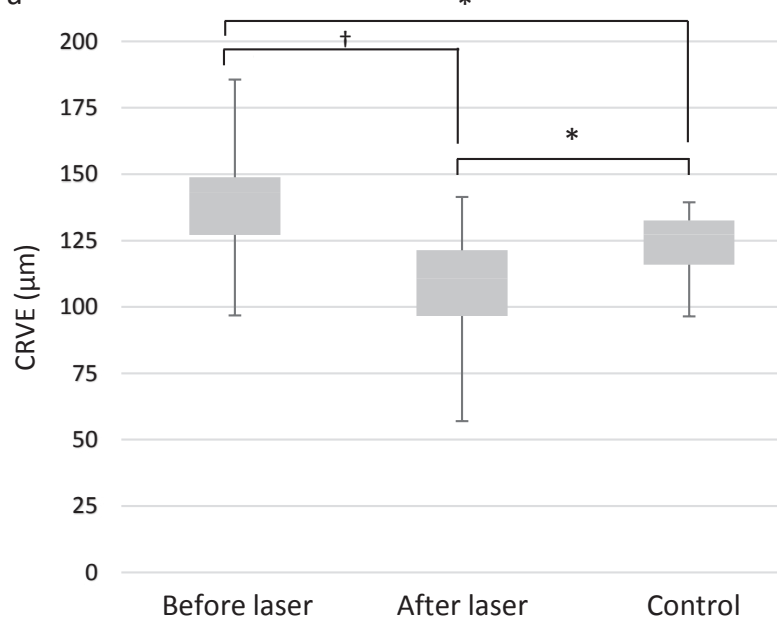

b

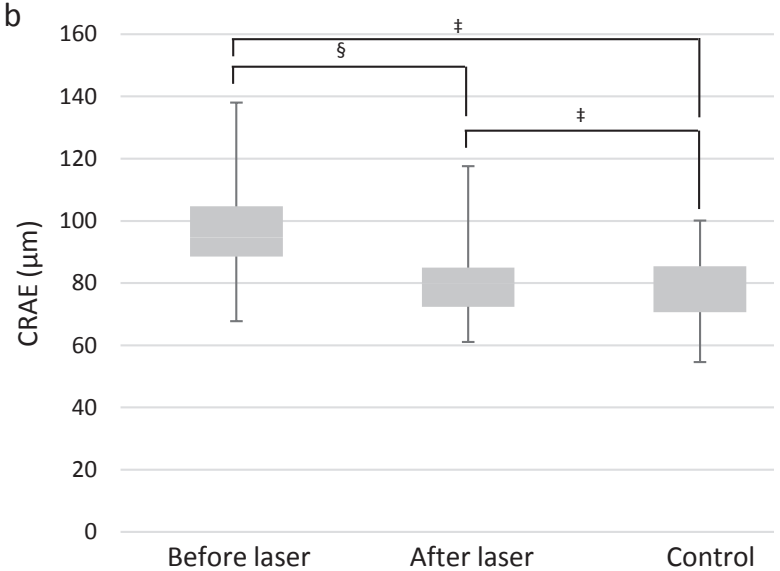

Figure 1. CRVE and CRAE ( $\mu \mathrm{m})$ before laser, after laser, and control measured by VAMPIRE manual annotation tool. Low CRVE and CRAE represents decrease in vessel and arteriole caliber, respectively. $C R V E=$ central retinal venular equivalent; $\mathrm{CRAE}=$ central retinal arteriolar equivalent; VAMPIRE=vessel assessment and measurement platform software for images of the retina

*Unpaired $t$-test, $p<0.001$; ${ }^{\dagger}$ paired $t$-test, $p<0.001$; ${ }^{\ddagger} M a n n-$ Whitney test, $p>0.05$; ${ }^{\S}$ Wilcoxon test, $p>0.05$

greater than control patient values $(p<0.001)$ and statistically significant difference between post-laser photocoagulation and control patient values. Figure $1 \mathrm{~b}$ shows the outcome of the treatment based on CRAE. No significant decrease of CRAE before and after laser photocoagulation.

\section{DISCUSSION}

This study showed that CRVE clinically increased in diabetic retinopathy compared with the control group. Several studies showed the widening of venous blood vessels in the retina. Chey and Park ${ }^{5}$ stated that the increased diameter of retinal blood vessels correlated with the prevalence and development of diabetic retinopathy, Jeganathan et al $^{16}$ reported that glucose level was associated with large retinal venular diameters in multiethnic Asian population; Liew et $\mathrm{al}^{17}$ noted that high hemoglobin $\mathrm{A} 1 \mathrm{C}$ and prolonged diabetes duration predicts greater venular vasodilation. In particular, the diameter of the central retinal vein correlates directly with retinopathy severity. Klein et al ${ }^{18}$ revealed that an increase in CRVE is associated with a high risk of diabetic retinopathy.

Tissue hypoxia triggers retinal vasodilatation. ${ }^{2}$ Retinal capillary leukocytosis occurs within weeks of diabetes onset; in this condition, blood-borne leucocytes adhere strongly to the endothelial plasma membrane, become entrapped, and cause capillary occlusion. This blockage in capillaries causes reduced blood flow in the retina and exacerbates local tissue ischemia. Hypoperfusion further contributes to low-grade, chronic inflammation of the retinal vasculature, resulting in further capillary dropout and the development of a progressive, irreversible hypoxia. Among the proposed mechanisms to explain retinal hypoxic vasodilatation are the release of metabolic factors from the surrounding neural tissues and the production of vasoactive agents from the endothelium. ${ }^{2}$

Furthermore, in our study, we observed that the caliber of the CRVE controls was $121.5 \mu \mathrm{m}$. In accordance with the research by Ding et al, ${ }^{19}$ the retinal vascular image consisted of arterioles and venules, with a diameter of about $100 \mu \mathrm{m}$. However, this finding differs with the results published from Sydney Childhood Eye Cohort, which stated that the average size of the caliber retinal arteriolar is $165.6 \mu \mathrm{m}$ and the venular caliber, $232.0 \mu \mathrm{m} .{ }^{20}$ We are unsure on how to explain these large variances between populations. However, such conditions may be related to the relative novelty of these measurements and the use of different algorithms.

The diameter of retinal venous decreased in patients with diabetic retinopathy following laser photocoagulation. ${ }^{21}$ This condition is consistent with the results of previous studies, and can be considered a supportive of the "oxygen theory." Vasoconstriction occurs in retinal branch vessels that respond to laser photocoagulation due to an increase in retinal oxygen pressure in the ischemic region. This phenomenon causes a decrease in intravascular stress in the capillaries and veins associated with passive 
vasoconstriction in accordance with Laplace's law. ${ }^{6}$ Laser photocoagulation has a regression effect of neovascularization, which can prevent and stop the progression of diabetic retinopathy. The laser beam used can cause small burns on the peripheral retina. This burn causes abnormal blood vessels to shrink and disappear. Meanwhile, approximately 6-8 weeks are needed for complete shrinkage of blood vessels. ${ }^{22,23}$

We assessed the comparison of CRAE in diabetic retinopathy (Figure $1 \mathrm{~b}$ ). The course of diabetic retinopathy obtained but arterial blood vessel dilation was $93 \pm 23.1 \mu \mathrm{m}$, whereas changes occurred in CRAE but in a less dramatic manner than that which occurred in the CRVE. The retinal arterioles control vascular resistance and hydrostatic pressure. The diameter of retinal arterioles is controlled by oxygen levels. The diameter of arterioles and venules in the retina is an indication of hydrostatic blood pressure in the retinal microcirculation. ${ }^{24}$ In Wisconsin Epidemiological Study of Diabetic Retinopathy with total sample of 1,098 diabetic patients who had received grading through fundus photos and computerized measurements of the CRAE and CRVE, the results indicate that CRVE changed to statistically significant degrees, but CRAE did not, which fits with our own results. Furthermore, CRVE was associated with the incidence and progression of diabetic retinopathy, but not CRAE. Our data were from a short-term prospective treatment response study, not a long-term epidemiological study. Thus, we cannot compare these data. Furthermore, Chey and Park ${ }^{5}$ showed that diabetic retinopathy patients have smaller retinal artery diameters and larger retinal vein diameter. This finding was possibly due to the broad relationship of CRVE with endothelial dysfunction, inflammatory changes, and hyperglycemia, which are related factors in the pathogenesis of diabetic retinopathy. ${ }^{5,18}$ Such conditions might have caused a better therapeutic response from CRVE compared with CRAE, which resulted in a more significant CRVE reduction than CRAE.

A limitation of our study is that the relatively small number of subjects due to loss to follow-up VAMPIRE, which requires manual annotation tool, can possibly introduce variations and loss of precision in these measures. However, this does not lead to a systematic bias because the same imprecision affects both preand post-laser images. Smoking was not entered as one of the exclusion criteria in this study overthought long-term effect of smoking on microcirculation may contribute to mixture between smoking and cardiovascular disease.

In conclusion, we have demonstrated that there is a significant decrease in vessel caliber (CRVE) after laser photocoagulation. Laser photocoagulation decreases retinal vascular caliber, even in the absence of change in the grade of diabetic retinopathy.

\section{Conflict of Interest}

The authors affirm no conflict of interest in this study.

\section{Acknowledgment}

The authors gratefully acknowledge the support of VAMPIRE team, University of Dundee, UK and support from Ninewells NHS Hospital and Medical School, Dundee, UK.

Funding Sources

None.

\section{REFERENCES}

1. Nguyen TT, Wang JJ, Wong TY. Retinal vascular changes in prediabetes and prehypertension: new findings and their research and clinical implications. Diabetes Care. 2007;30(10):2708-15.

2. Stitt AW, Curtis TM, Chen M, Medina RJ, McKay GJ, Jenkins A, et al. The progress in understanding and treatment of diabetic retinopathy. Prog Retin Eye Res. 2016;51:156-86.

3. Ding J, Wai KL, McGeechan K, Ikram MK, Kawasaki R, Xie J, et al. Retinal vascular caliber and the development of hypertension: a meta-analysis of individual participant data. J Hypertens. 2014;32(22):207-15.

4. Liew G, Wang JJ. Retinal vascular signs: a window to the heart? Rev Esp Cardiol. 2011;64(6):515-21. Spanish.

5. Chey JH, Park JM. Retinal vascular caliber changes in diabetic retinopathy after panretinal photocoagulation and additive bevacizumab injections. J Korean Ophthalmol Soc. 2016;57(6):917-23.

6. Maár N, Luksch A, Graebe A, Ergun E, Wimpissinger B, Tittl $M$, et al. Effect of laser photocoagulation on the retinal vessel diameter in branch and macular vein occlusion. Arch Ophthalmology. 2004;122(7):987-91.

7. Dashtbozorg B. Advanced image analysis for the assessment of retinal vascular changes [thesis]. [Porto]: University of Porto. 2015. p. 27-8.

8. Perez-Rovira A, Gillivray TM, Trucco E, Chin KS, Zutis K, Lupascu $C$, et al. VAMPIRE: Vessel Assessment and Measurement Platform for Images of the Retina. Conference proceedings: Annual International Conference of the IEEE Engineering in Medicine and Biology Society; 2011:3391-4.

9. Downie E, Tokarev J, Divani A, Koozekanani DD. Comparison of two free retinal vascular measurement software packages: IVAN and VAMPIRE. Invest Ophthalmol Vis Sci. 2015;56(7):3320.

10. Pead E, Megaw R, Cameron J, Fleming A, Dhillon B, Trucco E, et al. Automated detection of age-related macular degeneration in color fundus photography: a systematic review. Surv Ophthalmol. 2019;64(4):498-511.

11. MacGillivray TJ, McGrory S, Pearson T, Cameron J. Retinal imaging in early Alzheimer's disease. In: Perneczky R.,editor. Biomarkers for preclinical Alzheimer's disease. Neuromethods, vol 137. New York: Humana Press; 2018.

12. McGrory S, Cameron JR, Pellegrini E, Warren C, Doubal FN, Deary IJ, et al. The application of retinal fundus camera imaging in dementia: a systematic review. Alzheimers Dement. 2016;6:91-107. 
13. MacGillivray TJ, Trucco E, Cameron JR, Dhillon B, Houston JG, van Beek EJ. Retinal imaging as a source of biomarkers for diagnosis, characterization and prognosis of chronic illness or long-term conditions. Br J Radiol. 2014;87(1040):20130832.

14. Trucco E, Ruggeri A, Karnowski T, Giancardo L, Chaum E, Hubschman JP, et al. Validating retinal fundus image analysis algorithms: issues and a proposal. Invest Ophthalmol Vis Sci. 2013;54(5):3546-59.

15. Vampire [Internet]. [cited 2018 Nov 14]. Available from: https:// vampire.computing.dundee.ac.uk/index.html.

16. Jeganathan VS, Sabanayagam C, Tai ES, Lee J, Lamoureux E, Sun C, et al. Retinal vascular caliber and diabetes in a multiethnic Asian population. Microcirculation. 2009;16(6):534-43.

17. Liew G, Benitez-Aguirre $P$, Craig ME, Jenkins AJ, Hodgson $L A B$, Kifley $A$, et al. Progressive retinal vasodilation in patients with type 1 diabetes: a longitudinal study of retinal vascular geometry. Invest Ophthalmol Vis Sci. 2017;58(5):2503-9.

18. Klein R, Myers CE, Lee KE, Gangnon R, Klein BE. Changes in retinal vessel diameter and incidence and progression of diabetic retinopathy. Arch Ophthalmol. 2012;130(6):749-55.

19. Ding J, Ikram MK, Cheung CY, Wong TY. Retinal vascular calibre as a predictor of incidence and progression of diabetic retinopathy. Clin Exp Optom. 2012;95(3):290-6.

20. Ikram MK, Ong YT, Cheung CY, Wong TY. Retinal vascular caliber measurements: clinical significance, current knowledge and future perspectives. Ophthalmologica. 2013;229(3):125-36.

21. Grunwald JE, Riva CE, Brucker AJ, Sinclair SH, Petrig BL. Effect of panretinal photocoagulation on retinal blood flow in proliferative diabetic retinopathy. Ophthalmology. 1986;93(5):590-5.

22. Dijah, Iskandar E, Musa RI. Effectiveness of panretinal photocoagulation in treatment of diabetic retinopathy. Ophthalmol Ina. 2015;41:1.

23. McCannel CA, Atebara NH, Kim SJ, Leonard BC, Rosen RB, Sarraf D, et al. Retina and vitreous. American Academy of Ophthalmology. 2016, section 12:349-401.

24. Stefánsson E. The mechanism of retinal photocoagulation How does the laser work? EurOphthal. 2009;02(01):76-9. 